\title{
D-branes at Singular Curves of Calabi-Yau Compactifications
}

\author{
ILKA BRUNNER \\ Dept. of Physics and Astronomy, Rutgers University, \\ Piscataway, NJ 08855, U.S.A. \\ VOLKER SCHOMERUS \\ II. Inst. für Theoretische Physik, Universität Hamburg \\ Luruper Chaussee 149, D-22761 Hamburg, Germany
}

January 20, 2000

\begin{abstract}
We study the Gepner model description of D-branes in Calabi-Yau manifolds with singular curves. From a geometrical point of view, the resolution of singularities leads to additional homology cycles around which branes can wrap. Using techniques from conformal field theory we address the construction of boundary states for branes wrapping additional 3-cycles on the resolved Calabi-Yau manifold. Explicit formulas are provided for $\mathbb{Z}_{2}$ singular curves.
\end{abstract}

e-mail:ibrunner@physics.Rutgers.EDU; vschomer@x4u.desy.de 


\section{Introduction}

During the last years our understanding of non-perturbative aspects of string- and field theories has improved tremendously. In this process, the analysis of D-branes played a prominent role. While many of these investigations have been devoted to string theories in a flat background, it has been tried to extend this knowledge to curved backgrounds preserving fewer supersymmetries using various approaches. In this context, Calabi-Yau (CY) compactifications are of particular interest. There exist essentially two ways to approach $D$-branes in CY-manifolds: in the large radius limit we can describe them geometrically as branes wrapping around holonomy cycles $[1,2]$. It has been found that D-branes in a geometrical phase are naturally described in terms of K-theory classes [3, 4]. On the other hand, these geometrically intuitive concepts are not available in the stringy regime. Here, we can employ methods of boundary conformal field theory (CFT) instead to study D-branes at Gepner points $[5,6,7,8]$. The comparison of these two approaches has been initiated in [9] (see also $[10,11,12]$ for more recent work in this direction).

The present work focuses mainly on extending the CFT constructions of D-branes at the Gepner points of CY compactifications. While a large class of such D-branes was obtained in [5], a closer investigation of their RR-charges shows that none of them corresponds to branes wrapping the exceptional cycles which appear from the resolution of possible singularities. Since there are only a few non-singular cases (of which the quintic is the most prominent), one would certainly hope that CFT-techniques can provide additional D-branes for almost all Gepner models. This is the problem we are going to address below. In particular, we will construct so-called boundary states that are associated with branes wrapping the additional three-cycles induced by the resolution of a $\mathbb{Z}_{2}$-singularity over a curve in the CY-space.

D-branes in a flat space with orbifold singularities have been studied extensively in the string theory literature, starting with the work of Douglas and Moore [13]. In particular, it is known how to describe D-branes wrapping the collapsed cycles at the orbifold point. The main idea is to attach Chan-Paton factors to the ends of the open strings and define an orbifold action on them. Boundary states corresponding to branes away from the orbifold fixed points are obtained by summing over the brane's pre-images in the covering space. At the fixed points, however, the expressions for boundary states can involve contributions from twisted sectors of the theory, leading to a charge under RR-potentials coming from the twisted sector. We shall see these concepts reappearing in the non-geometrical CFT analysis.

Our analysis of D-branes in Gepner models will be formulated in the framework of simple current orbifolds. For closed string theories, the necessary techniques were developed long ago in $[14,15]$. Open string descendants of simple current orbifolds were investigated systematically by Fuchs and Schweigert [16, 17, 18, 19] and more recently in [20]. Some non-trivial examples have also been studied previously in [21, 22]. The general results show that the boundary states of [5] can be further 'resolved', if the action of an 
appropriate set $\Gamma$ of simple currents on conformal families of the theory possesses short orbits, i.e. orbits of length being less than the order $|\Gamma|$ of $\Gamma$.

Some general aspects of simple current orbifolds will be discussed in the next section. In particular, we shall explain how one can obtain a large set of D-brane states in the orbifold theory by an appropriate projection. For reasons to become clear later, the branes that result from this construction will be called untwisted D-branes. In Section 3 we illustrate the whole procedure at the example of A-type boundary states in Gepner models, thereby recovering precisely the A-type D-brane states listed in [5]. Geometrically, these correspond to branes wrapping middle dimensional cycles on the CY-manifold [2]. As we argue in Section 4, the untwisted D-branes do not wrap exceptional cycles. This motivates our search for additional D-brane states in Section 5. There we will show that untwisted D-branes at a $\mathbb{Z}_{2}$ singularity over some curve $C$ can be further resolved. Explicit formulas for the associated boundary states and the open string partition functions are provided. We finally conclude with a number of remarks on possible extensions. These include the analysis of B-type boundary states and of branes wrapping $\mathbb{Z}_{N^{-}}$singularities. While the present techniques do not suffice to resolve branes at $\mathbb{Z}_{N \neq 2}$-singularities, they can be used to study B-type boundary states and the comparison with the results of [5] provides new evidence for mirror symmetry in the open string sector.

\section{Orbifolds and untwisted D-branes}

The aim of this section is to review some results on simple current orbifolds and a general method for the construction of D-branes in the untwisted sector of the orbifold theory. This will be applied to Gepner models below.

\subsection{Simple current orbifolds - the bulk theory}

The simple current techniques developed in $[14,15]$ allow to construct new modular invariants from existing ones. A well-known class of examples is provided by Gepner models $[23,24]$, where the GSO-projected partition function is obtained using the spectral flow operator as a simple current. Other applications in string theory include the construction of $(0,2)$ models which lead to $\mathcal{N}=1$ space-time supersymmetric theories in four uncompactified dimensions [25].

In this subsection, we briefly summarize the main results of $[14,15]$. Consider some given bulk theory with a bosonic chiral algebra $\mathcal{W}$. We label classes of irreducible representations of $\mathcal{W}$ by labels $i, j, k$ taken from an index set $\mathcal{J}$. Within $\mathcal{J}$ we may find some non-trivial classes $g \in \mathcal{J}$ such that the fusion product of $g$ with any other $j \in \mathcal{J}$ gives again a single class $g \cdot j=g j \in \mathcal{J}$. Such classes $g$ are called simple currents and the set $\mathcal{C}$ of all these simple currents forms an abelian subgroup $\mathcal{C} \subset \mathcal{J}$. The product in 
$\mathcal{C}$ is inherited from the fusion product of representations. From now on, let us fix some subgroup $\Gamma \subset \mathcal{C}$.

Through the fusion of representations, the index set $\mathcal{J}$ comes equipped with an action $\Gamma \times \mathcal{J} \rightarrow \mathcal{J}$ of the group $\Gamma$ on labels $j \in \mathcal{J}$. Under this action, $\mathcal{J}$ may be decomposed into orbits. The space of these orbits will be denoted by $\mathcal{J} / \Gamma$ and we use the symbol $[j]_{\Gamma}$ to denote the orbit represented by $j \in \mathcal{J}$. By construction, the length of the orbit of the identity $1 \in \mathcal{J}$ is given by the order $|\Gamma|$ of the group $\Gamma$. Other orbits may be shorter since there can be fixed points, i.e. labels $j \in \mathcal{J}$ for which

$$
g \cdot j=j \quad \text { for some } \quad g \in \Gamma .
$$

The subgroup of all simple currents leaving some $j \in \mathcal{J}$ fixed is called the stabilizer of $j$ :

$$
\mathcal{S}_{j}=\{g \in \Gamma \mid g \cdot j=j\}
$$

Two labels $j_{1}, j_{2}$ possess isomorphic stabilizers, if they are on the same orbit, i.e. if there exists an element $g \in \Gamma$ such that $g \cdot j_{1}=j_{2}$.

For all simple currents $g \in \Gamma$ and all labels $j \in \mathcal{J}$ we define the monodromy charge $Q_{g}(j) \in \mathbf{R} / \mathbf{Z} \cong S^{1}$ of $j$ with respect to $g$ by

$$
Q_{g}(j):=h_{g}+h_{j}-h_{g \cdot j} \quad \bmod 1
$$

Here, $h_{l}$ denotes the non-integer part of the conformal dimension of the conformal primary that is associated with $l \in \mathcal{J}$. The meaning of $Q_{g}(j)$ can be easily understood once we choose two fields $J(z)$ and $\psi(w)$ from the conformal families $g \in \Gamma \subset \mathcal{J}$ and $j \in \mathcal{J}$, respectively. Their operator product will give fields $\psi^{\prime}(w)$ within a single conformal family $g \cdot j$, i.e.

$$
J(z) \psi(w) \sim(z-w)^{h_{J}+h_{\psi}-h_{\psi^{\prime}}} \psi^{\prime}(w)+\ldots .
$$

If we move $z$ once around $w$ we pick up some phase factor $\exp \left(2 \pi i Q_{g}(j)\right)$ which is given by the monodromy charge defined above. Let us finally note that the map $\exp (2 \pi i Q .(j))$ : $\Gamma \rightarrow S^{1}$ defined by $g \mapsto \exp \left(2 \pi i Q_{g}(j)\right)$ gives rise to a 1 -dimensional representation of the group $\Gamma$.

In the case that the simple currents have integer conformal weight, we can include them into the chiral algebra of the theory to form an extended chiral algebra $\mathcal{W}(\Gamma)$. Note that in this case the monodromy charge $Q_{g}(j)$ depends only on the equivalence class $[j]=[j]_{\Gamma}$ of $j \in J$ in the space $\mathcal{J} / \Gamma$ of orbits. An orbit $[j]$ is said to be invariant, if $Q_{g}([j])=Q_{g}(j)=0$ for all $g \in \Gamma$. We can now write down the partition function for the new orbifold theory. Under certain conditions that are satisfied for all cases to be discussed, conformal families of the extended algebra $\mathcal{W}(\Gamma)$ are labeled by pairs $\left([j]_{\Gamma}, \tau\right)$ of invariant orbits along with irreducible representations $\tau$ of the associated stabilizer subgroup, i.e. by $[j]_{\Gamma}$ with $Q_{\Gamma}([j])=0$ and $\tau: \mathcal{S}_{j} \rightarrow U(1)$. The new orbifold theory 
possesses a diagonal modular invariant partition function with respect to the extended algebra $W(\Gamma)$, i.e.

$$
Z^{\text {orb }}=\sum_{[j], Q_{\Gamma}([j])=0}\left|\mathcal{S}_{[j]}\right|\left|\sum_{g \in \Gamma / \mathcal{S}_{j}} \chi_{g j}\right|^{2}
$$

Characters appearing with a multiplicity given by the order of the stabilizer are associated with inequivalent representations of the larger algebra $\mathcal{W}(\Gamma)$. Upon restriction to the original chiral algebra $\mathcal{W}$, these representations become equivalent. The new partition function is obviously non-diagonal with respect to the smaller algebra $\mathcal{W}$.

\subsection{D-branes in the untwisted sector}

In this section we shall explain how to construct D-branes in the untwisted sector of simple current orbifolds. Starting from D-branes in the original theory we can obtain certain boundary states for the orbifold theory by a projection onto invariant sectors. This projection method was invented in [5] to get GSO invariant boundary states in Gepner models. The GSO projection can be understood through simple current extension or orbifolds. The methods of [5] (for A-type boundary states) can easily be extended to open descendants of other simple current modular invariants. The idea is to construct boundary states of the theory with a non-diagonal orbifold partition function from boundary states of the original diagonal theory.

So let us suppose we are given our original theory with a partition function

$$
Z=\sum_{j \in \mathcal{J}}\left|\chi_{j}\right|^{2}
$$

We are looking for associated theories on the half plane such that chiral fields obey the gluing condition $W(z)=(\Omega \bar{W})(\bar{z})$ all along the boundary $z=\bar{z}$ and for all chiral fields $W$ of the theory. For one special choice of the gluing automorphism $\Omega$ one can apply Cardy's prescription to find a complete set of elementary boundary states of the original theory (see e.g. [5] for more details). These are given by the formula

$$
\left.|I\rangle=\sum_{j} \frac{S_{I j}}{\sqrt{S_{0 j}}}|j\rangle\right\rangle
$$

Here, the element $|j\rangle\rangle$ is the generalized coherent ('Ishibashi'-) state associated with the sector $\mathcal{H}_{j} \otimes \overline{\mathcal{H}}_{j}$ of the theory. $S$ is the modular S-matrix of the rational theory under consideration.

Let us now consider the orbifold of the original theory obtained from the action $g \rightarrow$ $\exp \left(2 \pi i Q_{g}\right)$ of our abelian group $\Gamma$ on the state space $\mathcal{H}$. Here we think of the monodromy charge $Q_{g}$ as an operator on the space $\mathcal{H}=\oplus_{j} \mathcal{H}_{j} \otimes \overline{\mathcal{H}}_{j}$ that acts by multiplication with $Q_{g}(j)$ upon restriction to the subspaces $\mathcal{H}_{j} \otimes \overline{\mathcal{H}}_{j}$. As usual, the untwisted sector of the 
orbifold theory contains the states of the original theory that are invariant under the action of $\Gamma$. We can map the states from $\mathcal{H}$ to the untwisted sector of the orbifold theory with the help of the projector

$$
P^{0}=\frac{1}{|\Gamma|} \sum_{g \in \Gamma} \exp \left(2 \pi i Q_{g}\right) .
$$

The idea in [5] was to take the (appropriately rescaled) image of the Cardy boundary states (6) under $P^{0}$ as boundary states of the orbifold theory, i.e.

$$
\left.|I\rangle_{\text {proj }}=\kappa P^{0}|I\rangle=\frac{\kappa}{|\Gamma|} \sum_{g \in \Gamma} e^{2 \pi i Q_{g}} \sum_{j} \frac{S_{I j}}{\sqrt{S_{0 j}}}|j\rangle\right\rangle
$$

By construction, these states are linear combinations of generalized coherent states in the orbifold theory. Hence, it only remains to check that they give rise to consistent open string spectra. To this end one may exploit the following property of modular S-matrices

$$
S_{g I j}=e^{2 \pi i Q_{g}(j)} S_{I j}
$$

Insertion into our previous expression for the projected boundary states $|I\rangle_{\text {proj }}$ yields the interesting result

$$
|I\rangle_{\text {proj }}=\frac{\kappa}{|\Gamma|} \sum_{g \in \Gamma}|g I\rangle
$$

This means that in a general model, the charge projected boundary states are orbits of boundary states in the theory before orbifolding. The formula should be regarded as an algebraic analogue of the geometric prescription to sum over pre-images of a brane in the covering space. Hence, the open string spectra associated with these the boundary states $|I\rangle_{\text {proj }}$ are

$$
\begin{aligned}
Z_{I J}^{\text {proj }}(q) & :=\operatorname{proj}\left\langle\theta I\left|\tilde{q}^{\frac{1}{2}\left(L_{0}+\bar{L}_{0}\right)-\frac{c}{24}}\right| J\right\rangle_{\text {proj }} \\
& =\frac{\kappa^{2}}{|\Gamma|^{2}} \sum_{g, h \in \Gamma, k} N_{k}^{g I^{\vee} h J} \chi_{k}(q)=\frac{\kappa^{2}}{|\Gamma|} \sum_{g, k} N_{k}^{g I^{\vee} J} \chi_{k}(q) .
\end{aligned}
$$

Here, $\theta$ denotes the CPT operator of the bulk theory and $I^{\vee}$ is the label conjugate to $I$ (see [5] for details). If we choose

$$
\kappa=\sqrt{|\Gamma|}
$$

then the coefficients of the characters on the right hand side are guaranteed to be integer. But they may still possess a common divisor. This happens whenever $I$ or $J$ is fixed under the action of some element $g \in \Gamma$. If one of the labels is on such a short orbit, then the sum in (9) consists of several equal terms. For $I=J$, the number of equal terms is given by the order $\left|\mathcal{S}_{I}\right|$ of the stabilizer subgroup $\mathcal{S}_{I} \subset \Gamma$ of $I$. We shall see below that 
in the orbifold theory there exist $\left|\mathcal{S}_{I}\right|$ boundary states associated with the label $I$ which differ by a representation of the stabilizer subgroup $\mathcal{S}_{I}$. The projection analysis provides one linear combination of these $\left|\mathcal{S}_{I}\right|$ states which contains only contributions from the untwisted sector.

\section{Gepner models and untwisted D-branes}

The aim now is to apply the general theory outlined above to an important class of examples, namely to Gepner models [23, 24] (see also [26] for a review). These are exactly solvable CFTs which are used to study strings moving on a Calabi-Yau manifold at small radius [27]. Their construction employs an orbifold-like projection starting from a tensor products of $r \mathcal{N}=2$ minimal models. In our presentation we shall assume that there are $d=1$ complex, transverse, external dimensions in light cone gauge and that the number $r$ of minimal models equals $r=5$.

\subsection{The building blocks of Gepner models}

Our main building blocks are $\mathcal{N}=2$ minimal models at level $k$. These are SCFTs with central charge $c=\frac{3 k}{k+2}<3[28,29,30,31]$. One can label primaries of the bosonic subalgebra by 3 integers, $(l, m, s)$ taking values in the range

$$
0 \leq l \leq k ; \quad 0 \leq|m-s| \leq l ; s \in\{-1,0,1\} ; l+m+s=0 \bmod 2 .
$$

In many respects, $l$ and $m$ behave like the familiar labels in an $S U(2)_{k}$ WZW model. The third label $s$ determines the spin structure. States with $s=0$ are in the NS sector while $s= \pm 1$ correspond to the two chiralities in the $R$ sector. The conformal weights and $U(1)$ charges of these primary fields can be computed by means of the formulas

$$
\begin{aligned}
& h_{m, s}^{l}=\frac{l(l+2)-m^{2}}{4(k+2)}+\frac{s^{2}}{8} \bmod 1, \\
& q_{m, s}^{l}=\frac{m}{k+2}-\frac{s}{2} \bmod 2 .
\end{aligned}
$$

There are some distinguished labels $(l, \pm l, 0) \in \mathcal{J}$ in the NS sector which are associated with the $\mathcal{N}=2$ (anti-) chiral primaries. They play a special role in the Landau-Ginzburg description of minimal models where they are identified with powers $X^{l} \sim(l, l, 0)$ of the Landau-Ginzburg field $X$.

Our set $\mathcal{J}$ of conformal families contains triples $(l, m, s)$ from the standard range. It will be rather convenient below to consider an extended set $\tilde{\mathcal{J}}$ of labels $(l, m, s) \in$ $\{0, \ldots, k\} \times \mathbb{Z}_{2 k+4} \times \mathbb{Z}_{4}$ with the only additional constraint $l+m+s=$ even. For each label $(l, m, s) \in \mathcal{J}$ there exist exactly two labels in $\tilde{\mathcal{J}}$, namely $(l, m, s)$ and $(k-l, m+k+$ 
$2, s+2)$. In other words, the extended set $\tilde{\mathcal{J}}$ carries an action of $\mathbb{Z}_{2}$ that maps $(l, m, s)$ to $(k-l, m+k+2, s+2)$ such that our original set $\mathcal{J}$ is simply the quotient $\tilde{\mathcal{J}} / \mathbb{Z}_{2}$. Passing from $\tilde{\mathcal{J}}$ to $\mathcal{J}$ is known as field identification.

The simple currents of an $\mathcal{N}=2$ minimal model can be determined from the fusion rules. For the label $l$ these are given by the usual $S U(2)$ fusion rules while both other labels add like representations of the abelian groups $\mathbb{Z}_{2 k+4}$ and $\mathbb{Z}_{4}$, respectively. This implies that e.g. $(0,1,1)$ and $(0,0,2)$ are both simple currents. They are of special interest in the context of Gepner models and will be used to generate our simple current group $\Gamma .(0,1,1)$ is the spectral flow by $1 / 2$ unit and $(0,0,2)$ the world-sheet supersymmetry generator. $(0,0,2)$ is a simple current of order 2 and can be used to group the world-sheet fields into supermultiplets. The order of the simple current $(0,1,1)$ is model dependent. To see this, we apply the current $2 k+4$ times to the identity. This will lead us back to the identity whenever the level $k$ is even. Since $(0,0,2)$ is nowhere on this orbit, $(0,0,2)$ and $(0,1,1)$ togther generate the simple current group $\Gamma=\Gamma_{k}=\mathbb{Z}_{2 k+4} \times \mathbb{Z}_{2}$ for even level $k$. When $k$ is odd, however, we reach the field $(0,0,2)$ after $2 k+4$ applications of $(0,1,1)$ and hence we have to apply the simple current $(0,1,1)$ another $2 k+4$ times. In this case, the orbit contains the label $(0,0,2)$ and hence our orbifold group is $\Gamma=\Gamma_{k}=\mathbb{Z}_{4 k+8}$ for odd $k$.

Let us briefly describe the orbits for the action of $\Gamma_{k}$ on the set $\mathcal{J}$. Again, we need to treat the cases of even and odd $k$ separately. If $k$ is odd, the orbifold group $\Gamma_{k}$ acts freely so that all orbits have length $4 k+8$. For even level $k$, however, we generate short orbits of length $2 k+4$ whenever we start from a field $(l, m, s)$ with $l=k / 2$ because the label $l=k / 2$ is invariant under field identification. The stabilizer for these short orbits is a subgroup $\mathbb{Z}_{2} \subset \Gamma_{k}$.

From formula (13) and the definition relation (3) we can compute the monodromy charge:

$$
Q_{(0, \mu, \sigma)}^{k}(l, m, s):=\frac{m \mu}{2 k+4}-\frac{s \sigma}{4} \bmod 1 \quad \text { for all } \quad(0, \mu, \sigma) \in \Gamma_{k}
$$

Obviously, this expression defines a representation of our orbifold group $\Gamma_{k}$ for any choice of $(l, m, s) \in \mathcal{J}$.

$\mathcal{N}=2$ characters and their modular properties are described e.g. in [23, 24]. The characters $\chi_{l, m, s}$ of the representations listed above can be indexed by arbitrary labels $(l, m, s)$ in the extended set $\tilde{\mathcal{J}}$. In fact, we can use the field identification to define $\chi_{l, m, s}$ for $(l, m, s)$ outside the standard range (12) by $\chi_{m, s}^{l}=\chi_{m+k+2, s+2}^{k-l}$. For the modular S-matrix one has the explicit formula

$$
S_{(l, m, s),\left(l^{\prime}, m^{\prime}, s^{\prime}\right)}^{k}=\frac{1}{\sqrt{2}(k+2)} \sin \pi \frac{(l+1)\left(l^{\prime}+1\right)}{k+2} e^{i \pi m m^{\prime} /(k+2)} e^{-i \pi s s^{\prime} / 2}
$$

It is easy to check that $S$ satisfies the relation (8) for all $g \in \Gamma_{k}$. 
In addition to the minimal models, Gepner's constructions involve fermions from the external space-time sector of the theory. Their bosonic subalgebra is an $S O(2)_{1}$ current algebra. Its representations are labeled by $s=0, \pm 1,2 \in \mathbb{Z}_{4}$ and they possess the obvious abelian fusion rules. All sectors in this theory are simple currents and their monodromy charge is given by

$$
Q_{\sigma}^{\mathrm{f}}(s):=-\frac{s \sigma}{4} \bmod 1 \quad \text { for } \quad \sigma=0, \pm 1,2 .
$$

The property (8) is obeyed by the modular S-matrix $S_{s, s^{\prime}}^{\mathrm{f}}=(1 / 2) \exp \left(-i \pi s s^{\prime} / 2\right)$ of the $S O(2)_{1}$ current algebra.

\subsection{Gepner models in the bulk}

Let us now consider a tensor product of $r=5$ minimal models with levels $k_{i}, i=1, \ldots, r$ such that their central charges adds up to $c=9$. To get a full string theory, one needs to add a sector containing ghosts and a level $k=1$ current algebra that comes with the space-time sector. These tensor products do not give consistent string backgrounds with 4d spacetime SUSY. But there exists some orbifold theory of this tensor product theory that satisfies all the requirements of a consistent string background. For its description we need further notations. Let us introduce the following vectors

$$
\lambda:=\left(l_{1}, \ldots, l_{r}\right) \quad \text { and } \quad \mu:=\left(s_{0} ; m_{1}, \ldots, m_{r} ; s_{1}, \ldots, s_{r}\right)
$$

to label the tensor product of representations $\left(l_{j}, m_{j}, s_{j}\right)$ of the individual minimal models and of the representations $s_{0}=0,2, \pm 1$ that come with the level $k=1$ current algebra. The associated product of characters $\chi_{m_{i}, s_{i}}^{l_{i}}$ and $\chi_{s_{0}}$ is denoted by $\chi_{\mu}^{\lambda}(q)$.

Next, we introduce the special $(2 r+1)$-dimensional vectors $\beta_{0}$ with all entries equal to 1 , and $\beta_{j}, j=1, \ldots, r$, having zeroes everywhere except for the 1 st and the $(r+1+j)$ th entry which are equal to 2 . These vectors stand for particular elements in the group $\mathbb{Z}_{4} \times \prod_{i} \Gamma_{k_{i}}$. It is easily seen that they generate a subgroup $\Gamma=\mathbb{Z}_{K} \times \mathbb{Z}_{2}^{r}$ where $K:=$ $\operatorname{lcm}\left(2 k_{j}+4\right)$. Elements of this subgroup will be denoted by $\boldsymbol{\nu}=\left(\nu, \nu_{1}, \ldots, \nu_{r}\right)$. The monodromy charge of a pair $(\lambda, \mu)$ is

$$
Q_{\nu}(\lambda, \mu)=\nu \beta_{0} \cdot \mu+\sum_{i=1}^{r} \nu_{i} \beta_{i} \cdot \mu \bmod 1
$$

$$
\text { where } \begin{aligned}
\beta_{0} \cdot \mu & :=-\frac{s_{0}}{4}-\sum_{j=1}^{r} \frac{s_{j}}{4}+\sum_{j=1}^{r} \frac{m_{j}}{2 k_{j}+4}, \\
\beta_{j} \cdot \mu & :=-\frac{s_{0}}{2}-\frac{s_{j}}{2} .
\end{aligned}
$$


The orbifold group $\Gamma$ acts on the labels $\lambda$ and $\mu$ in the obvious way. There appear orbits of maximal length $K 2^{r}$ and short orbits of length $K 2^{r-1}$. The latter are characterized by the property that $\lambda=\left(l_{1}, \ldots, l_{r}\right)$ satisfy $l_{i}=k_{i} / 2$ for all $i$ such that $2 k_{i}+4$ is not a factor in $K / 2$.

As we mentioned before, the complete construction requires to include ghosts. Since the ghost sector will not play an important role in the later sections, we shall constrain ourselves to some brief remarks that are necessary in understanding Gepner models from an orbifold point of view.

When we consider the full theory, the field generating the $\mathbb{Z}_{K}$-symmetry contains a factor from the ghost sector and the space-time part of the spin field $S^{\alpha}$. In the $\pm 1 / 2$ picture, the operator can be represented as

$$
U(z)=e^{ \pm i \frac{\phi}{2}} e^{ \pm i \frac{1}{2} \sqrt{\frac{c}{3}} X} S_{\alpha}
$$

Here, $\phi$ denotes the bosonized super-ghost and we introduced the bosonic field $X$ whose derivative gives the $\mathrm{U}(1)$ current $J(z)=\sum_{i=0}^{r} J_{i}(z)$ in the tensor product of the $S O(2)_{1}$ theory with the minimal models. The precise relation is $J(z)=i \sqrt{\frac{c}{3}} \partial X$.

The operator (19) is a simple current and its internal part agrees with the simple current $\beta_{0}$ in the tensor product considered above. Since the operator (19) has total weight one, it can be added to the chiral algebra and we can use the formula (5) to determine the partition function of the orbifold theory. The formula requires to determine invariant orbits, i.e. orbits with vanishing monodromy charge. Taking the OPE of the spectral flow (19) with a vertex operator that represent space-time scalars of the theory gives the monodromy charge

$$
\tilde{Q}_{\nu}(\lambda, \mu)=\nu\left(\frac{\beta_{0} \cdot \mu}{2}+\frac{1}{2}\right)+\sum_{i=1}^{r} \nu_{i} \beta_{i} \cdot \mu \bmod 1
$$

by comparison with the general formula (4). $\tilde{Q}$ effectively replaces the monodromy charge $Q$ introduced in eq. (16). The orbits of vanishing monodromy charge are those of odd integer $U(1)$ charge.

To write a partition function of physical states one has to extract the physical degrees of freedom. This can be done by a projection onto light-cone variables which removes, in particular, the ghost sector. In practice, the light-cone degrees of freedom may be read off directly in the canonical ghost pictures.

An important reason to include ghosts is that the fields of the theory aquire the right commutation properties. One would like to incorporate this feature in the physical theory. Therefore, in the partition function, the fields are counted with a ghost-charge dependent phase factor $\exp \left(2 \pi i q_{\text {ghost }}\right)$. This means that the states with half-integer ghost charge, i.e. the RR-states, contribute with a negative sign.

Having discussed all aspects of the ghosts, which are relevant to the simple current construction of the Gepner partition function, we will discard them from now on and 
consider only the physical (light-cone) degrees of freedom. We are prepared to write down the partition function for a Gepner model describing a superstring compactification to 4 dimensions. It is given by

$$
Z_{G}^{(r)}(\tau, \bar{\tau})=\frac{1}{2} \frac{(\operatorname{Im} \tau)^{-2}}{|\eta(q)|^{2}} \sum_{\lambda, \mu ; \tilde{Q}(\lambda, \mu)=0} \sum_{\nu, \nu_{j}}(-1)^{\nu} \chi_{\mu}^{\lambda}(q) \chi_{\mu+\nu \beta_{0}+\nu_{1} \beta_{1}+\ldots+\nu_{r} \beta_{r}}^{\lambda}(\bar{q})
$$

The sign is the usual one occurring in (space-time) fermion one-loop diagrams. The $\tau$ dependent factor in front of the sum accounts for the free bosons associated to the 2 physical transversal dimensions of flat external space-time, while the $1 / 2$ is simply due to the field identification mentioned above. Except for these modifications, the formula for $Z_{G}$ is the same as eq. (5). Elements $g=\nu \beta_{0}+\ldots \nu_{r} \beta_{r}$ of the orbifold group $\Gamma$ are labeled by $\nu, \nu_{i}$ so that the second sum is over the full group $\Gamma$. Short orbits appear twice in the summation and give rise to an extra factor of 2 which is the order of the corresponding stabilizer subgroup. Since our orbifold group $\Gamma$ is abelian, we used additive notation for the action of elements $g \in \Gamma$ on the labels $\lambda, \mu$.

\subsection{Untwisted D-branes in Gepner models}

Using the general formalism outlined in Section 2.2 one can find a large set of boundary states [5] which respect the $\mathcal{N}=2$ world-sheet algebras of each minimal model factor of the Gepner model separately. To this end we start with Cardy boundary states of the tensor product theory. They are given by the expression (6) along with the formula (15) for the modular S-matrices of minimal models and the simple expression for the S-matrix of $S O(2)_{1}$ that we spelled out before. The generalized coherent states $\left.|j\rangle\right\rangle$ are now parametrized by pairs $j=(\lambda, \mu)$. Cardy's boundary states belong to some gluing condition $W(z)=\Omega \bar{W}(\bar{z}), z=\bar{z}$, which becomes $J_{i}(z)=-\bar{J}_{i}(\bar{z})$ on the $\mathrm{U}(1)$-currents of the individual theories. This means that they are A-type boundary conditions in the sense of $[2]$.

The boundary states $|I\rangle=:|\Lambda, \Xi\rangle$ we have just described depend on a spin vector $\Lambda=\left(L_{1}, \ldots, L_{r}\right)$ and a charge vector $\Xi=\left(S_{0} ; M_{1}, \ldots, M_{r} ; S_{1}, \ldots, S_{r}\right)$. From these states in the tensor product theory we can pass to boundary states of the Gepner model using the general strategy explained in Section 2.2. The projected boundary states in the orbifold theory are given by (see eqs. $(9,11)$ )

$$
|\Lambda, \Xi\rangle_{\text {proj }}=\frac{1}{\sqrt{K 2^{r}}} \sum_{\nu, \nu_{i}}(-1)^{\nu}(-1)^{\frac{\hat{s}_{0}^{2}}{2}}\left|\Lambda, \Xi+\nu \beta_{0}+\nu_{1} \beta_{1}+\ldots+\nu_{r} \beta_{r}\right\rangle .
$$

Here, the element $\hat{s}_{0}$ is an operator acting on closed string states which measures the value $s_{0}$. The whole factor $(-1)^{\hat{s}_{0}^{2} / 2}$ is needed to guarantee that in the open string partition function (similar to the closed string partition function) fields are counted with a phase 
factor referring to their ghost charge. If we insert the formula (6) with the appropriate modular S-matrix on the right hand side, we obtain the expressions established in [5],

$$
\left.|\alpha\rangle\rangle:=|\Lambda, \Xi\rangle\rangle_{\text {proj }}=\sum_{\lambda, \mu ; \tilde{Q}(\lambda, \mu)=0}(-1)^{\frac{s_{0}^{2}}{2}} B_{\alpha}^{\lambda, \mu}|\lambda, \mu\rangle\right\rangle .
$$

with the coefficients:

$$
B_{\alpha}^{\lambda, \mu}=\frac{\sqrt{K 2^{r}}}{2} e^{-i \pi \frac{s_{0} S_{0}}{2}} \prod_{j=1}^{r} \frac{1}{\sqrt{\sqrt{2}\left(k_{j}+2\right)}} \frac{\sin \left(l_{j}, L_{j}\right)_{k_{j}}}{\sqrt{\sin \left(l_{j}, 0\right)_{k_{j}}}} e^{i \pi \frac{m_{j} M_{j}}{k_{j}+2}} e^{-i \pi \frac{s_{j} S_{j}}{2}} .
$$

Here $\left(l, l^{\prime}\right)_{k}=\pi(l+1)\left(l^{\prime}+1\right) /(k+2)$. For these A-type boundary states the Ishibashi states are built on diagonal primary states, i.e. states in the untwisted sector, in accordance with our general theory in Section 2. The associated partition functions (10) aquire the following form (see also [5]):

$$
\begin{aligned}
Z_{\tilde{\alpha} \alpha}^{A}(q)=\frac{1}{2} \sum_{\lambda^{\prime}, \mu^{\prime}} & \sum_{\nu=0}^{K-1} \sum_{\nu_{i}=0,1}(-1)^{s_{0}^{\prime}+S_{0}-\tilde{S}_{0}} \delta_{s_{0}^{\prime}-\tilde{S}_{0}+S_{0}+\nu+2 \sum \nu_{i}-2}^{(4)} \\
& \times \prod_{j=1}^{r} N_{L_{j}, \tilde{L}_{j}}^{l_{j}^{\prime}} \delta_{\nu+M_{j}-\tilde{M}_{j}+m_{j}^{\prime}}^{\left(2 k_{j}+4\right)} \delta_{s_{j}^{\prime}-\tilde{S}_{j}+S_{j}+\nu+2 \nu_{j}}^{(4)} \chi_{\mu^{\prime}}^{\lambda^{\prime}}(q)
\end{aligned}
$$

The factor $1 / 2$ in front of the right hand side accounts for the fact that field identification causes each character to appear twice when we sum over $\lambda^{\prime}, \mu^{\prime}$ taken from the extended range.

If the two boundary conditions $\alpha, \tilde{\alpha}$ appearing in eq. (22) are both labeled by monodromy invariant orbits, they give rise to a monodromy invariant open string spectrum, i.e. to a spectrum that contains only odd-integer charges. One should recall, however, that non-invariant orbits of $\Gamma$ are also admissible as labels for boundary conditions. The condition for a supersymmetric open string spectrum consisting of monodromy invariant states is that the $\mathrm{U}(1)$ charge of the two orbits labeling the boundary conditions $\alpha$ and $\tilde{a}$ differs by an even integer.

\section{Singular curves on Calabi-Yau 3-folds}

The boundary states (20) we have constructed so far are only charged under $(c, c)$-fields in the untwisted sector. Typically, there exist additional $(c, c)$-fields in the twisted sectors of Gepner models. Their appearance is related to singularities of the associated Calabi-Yau spaces. The aim of this section is to explain this relation in some more detail. Once this is understood, it motivates the search for additional boundary states that are charged under 
fields in the twisted sectors. This will be addressed in the next section. Our presentation here will be rather sketchy. The interested reader can find more explanations and details e.g. in $[32,33,26,34,35]$.

Suppose we are given a Gepner model composed from $r=5$ minimal models with levels $\left(k_{1}, \ldots, k_{5}\right)$ chosen such that their central charges add up to $c=9$. Let us define integers $\omega_{i}=K /\left(2 k_{i}+4\right)$ where $K=\operatorname{lcm}\left(2 k_{i}+4\right)$ as before. Assuming that the Gepner model has an A-type modular invariant partition function, as we did before, we associate with it the following space $M=P_{\left(\omega_{1}, \ldots, \omega_{5}\right)}\left[\frac{K}{2}\right]$ which is defined by the equation

$$
M: \quad z_{1}^{k_{1}+2}+\ldots z_{5}^{k_{5}+2}=0
$$

evaluated in a weighted projective space where $\left(z_{1}, \ldots, z_{5}\right) \sim\left(\lambda^{\omega_{1}} z_{1}, \ldots, \lambda^{\omega_{5}} z_{5}\right)$ for $\lambda \in \mathbb{C}^{*}$. It is well known that the $(c, c)$-fields with total left- and right-moving charges $q, \bar{q}$ satisfying $q=1=\bar{q}$ correspond to harmonic $(2,1)$-forms on $M$. We denote the space of these forms by $H^{2,1}$.

Typically, the space $M$ possesses singularities which can be either singular points or singular curves. In string theory these singularities are resolved. Desingularization of singular curves provides a non-vanishing contribution to the Hodge number $h^{21}=\operatorname{dim} H^{2,1}$. We will make a more quantitative statement momentarily after a brief description of the possible singularities.

A singular curve on $M$ exists whenever three of the numbers $\omega_{i}$ have some non-trivial factor in common. To be specific, we suppose that these are $\omega_{3}, \omega_{4}, \omega_{5}$ and we denote their largest common divisor by $N$. Now our weighted projective space carries an action of $\mathbb{Z}_{N}$ defined by $\left(z_{1}, \ldots, z_{5}\right) \rightarrow\left(\eta^{\omega_{1}} z_{1}, \ldots, \eta^{\omega_{5}} z_{5}\right)$ where $\eta$ is some $N^{\text {th }}$ root of unity. By our choice of $N, \eta^{\omega_{i}}=1$ for $i=3,4,5$ and hence, there is a two complex dimensional subspace in the weighted projective space that remains fixed under the action of $\mathbb{Z}_{N}$. This subspace is parametrized by classes of $\left(0,0, z_{3}, z_{4}, z_{5}\right)$. The surface $M$ intersects with this singularity along the curve given by the equation

$$
C: z_{3}^{k_{3}+2}+z_{4}^{k_{4}+2}+z_{5}^{k_{5}+2}=0
$$

Putting all this together we see that $M$ possesses a $\mathbb{Z}_{N}$ singularity over each point of the curve $C$. At a generic point, the space transverse to the singular curve looks like the quotient $\mathbb{C}^{2} / \mathbb{Z}_{N}$ where $\mathbb{Z}_{N}$ acts on points $\left(w_{1}, w_{2}\right) \in \mathbb{C}^{2}$ by $\left(w_{1}, w_{2}\right) \rightarrow\left(\eta w_{1}, \eta^{-1} w_{2}\right)$.

The singularity at the origin of $\mathbb{C}^{2} / \mathbb{Z}_{N}$ is known as a rational double point of type $A_{N-1}$. It is resolved by gluing in a chain of $N-1$ projective spaces $\mathbb{P}^{1}$ which intersect pairwise transversely in one point and have self-intersection numbers -2 . In other words, the intersection matrix equals the negative Cartan matrix for an $A_{N-1}$ Dynkin diagram. When we resolve the $\mathbb{Z}_{N}$ singularity along the whole fixed curve $C$, we obtain locally a product of the curve $C$ and the chain of spheres we have just described. The resolution of a single fixed curve $C$ shifts the Hodge number $h^{21}$ by $(N-1) g$ with $g$ being the genus 
of the curve $C$ [36]. This can be understood by sending a one-cycle of the curve $C$ to the three-cycle swept out by the spheres fibered over the one-cycle [37]. (See also [33] for a discussion of singular curves in the context of gauge symmetry enhancement in type II theories.)

Let us turn back to the Gepner models and compare what we have learned about the resolution of singularities with the appearance of $(c, c)$-fields in the twisted sectors. As we have pointed out before, the Landau-Ginzburg description of minimal models involves an identification of the coordinate $z_{i}$ with the chiral primary field $\Phi_{m, s}^{i ; l}=\Phi_{1,0}^{i ; 1}$ in the $i^{\text {th }}$ minimal model. The subgroup $\mathbb{Z}_{K} \subset \Gamma$ acts on the latter by multiplication with the phase $\exp \left(2 \pi i /\left(2 k_{i}+4\right)\right)$. Note that the number $N$ must be contained as a factor in $K$ since each of the numbers $\omega_{3}, \omega_{4}, \omega_{5}$ is contained in $K$. Hence, we obtain an action of $\mathbb{Z}_{N} \subset \mathbb{Z}_{K}$ by

$$
\Phi_{1,0}^{i ; 1} \rightarrow e^{\frac{2 \pi i}{2 k_{i}+4} \frac{K}{N}} \Phi_{1,0}^{i ; 1}=e^{\frac{2 \pi i}{N} \omega_{i}} \Phi_{1,0}^{i ; 1}=\eta^{\omega_{i}} \Phi_{1,0}^{i ; 1}
$$

where $\eta=\exp (2 \pi i / N)$. This is precisely the transformation law of the coordinate $z_{i}$ under the action of the $\mathbb{Z}_{N}$ that is responsible for the singularity in the weighted projective space. Hence, we may identify the subgroup $\mathbb{Z}_{N} \subset \mathbb{Z}_{K} \subset \Gamma$ of the orbifold group with the geometrically acting group on our Calabi-Yau manifold $M$.

Combining the last paragraph with the previous discussion we can now formulate the relation between the singularities and $(c, c)$-fields more precisely [34, 35]. In fact, we expect to find $g$ of the $(c, c)$-fields with $q=1=\bar{q}$ in each of the $\nu=0 \bmod K / N$-twisted sectors. As there exist $N-1$ such sectors (the untwisted sector $\nu=0$ is not included), the number of new $(c, c)$-fields matches the shift of the Hodge number that we attributed to the desingularization of a single $\mathbb{Z}_{N}$-singular curve $C$.

While these new $(c, c)$-fields possess the same total right- and left moving $\mathrm{U}(1)$-charge, i.e. $q=\bar{q}$ by construction, the $\mathrm{U}(1)$-charges in the individual minimal models can be different. This may be seen from the obvious property $q^{(i)}-\bar{q}^{(i)}=\nu /\left(k_{i}+2\right) \bmod 1$ of fields in the $\nu$-twisted sector (it holds for $\nu_{i}=0$ ). The right hand side of these equations is non-zero unless $2 \nu$ can be devided by all the $2 k_{i}+4$. But this requires $2 \nu=K$ and hence we conclude that only the new $(c, c)$-fields in the $\nu=K / 2$-twisted sector have equal left- and right-moving charges $q^{(i)}=\bar{q}^{(i)}$ in all the individual minimal models. It is easy to see that all such fields lie on short orbits of our orbifold group $\Gamma$.

The boundary states we discuss in this work satisfy A-type boundary conditions in each minimal model rather than A-type boundary conditions for the diagonally embedded superconformal algebra only. This means that generalized coherent states in our boundary states are necessarily based on primary fields satisfying the condition $q^{(i)}=\bar{q}^{(i)}$. Hence, all we can hope for in the following is to find boundary states which are charged under the new $(c, c)$-fields in the $\nu=K / 2$-twisted sector, i.e. under the $(c, c)$-fields that arise from resolving a $\mathbb{Z}_{2}$-singularity along a fixed curve $C$. 
Example: The degree eight Fermat $\mathrm{CY}$ space $\mathbb{P}_{1,1,2,2,2}[8]$ corresponds to the choice $(k=6)^{2}(k=2)^{3}$ for the minimal models used in Gepner's construction. The surface has only one $\mathbb{Z}_{2}$ singularity over a curve $C$ of genus $g=3$. Resolving this singularity shifts the Hodge number $h^{21}$ by 3 . It is not difficult to list all the $(c, c)$-fields with $q=1=\bar{q}$ in this case. Except from those in the untwisted sector, there appear 3 additional such fields in the $\nu=8$-twisted sector with labels for their left-movers being given by

$$
(3,3,0) \times(3,3,0) \times(1,1,0) \times(0,0,0) \times(0,0,0)
$$

and by similar expressions obtained through a permutation of the last three factors. Obviously, these fields are all on short orbits of the group $\Gamma=\mathbb{Z}_{16} \times \mathbb{Z}_{2}^{5}$.

\section{Twisted D-branes in Gepner models}

We have argued in the previous section that a typical Gepner model has $(c, c)$-fields in the twisted sectors. Geometrically, this situation corresponds to the resolution of singularities inherited from the embedding projective space. D-branes can wrap the extra homology cycles on the resolved manifold. Therefore, we expect that there are boundary states containing Ishibashi states built on the $(c, c)$ fields in twisted sectors of the models. This is necessary in order to have D-branes which are charged under all the possible massless RR potentials in the theory. Since locally the singularities we shall be concerned with look like $\mathbb{Z}_{2}$ orbifolds of flat space, we will first review the construction of boundary states for fractional branes in flat space. This will help to motivate the procedure that allows to find twisted sector boundary states in Gepner models.

\subsection{Twisted boundary states in flat space}

D0 branes at orbifold singularities of flat space have been discussed from the open string point of view in [13]. There are different types of branes depending on the representation of the orbifold group on the Chan-Paton factors. The group $\mathbb{Z}_{2}$ has two inequivalent irreducible one-dimensional representations which are distinguished by a sign. These two representations lead to two types of fractional branes. In fact, to each of the two representations one can associate a boundary state [38, 39, 40, 41] (see also [42] for a related construction in a different context) for branes sitting at the origin $z=\left(z_{1}, z_{2}\right)=$ $(0,0)$. They are given by

$$
\left.|D(z=0) ; \pm\rangle=\frac{1}{\sqrt{2}}|D(z=0)\rangle \pm \sqrt{2}|D(z=0)\rangle\right\rangle^{\mathrm{tw}} .
$$

Here, $|D(z=0)\rangle\rangle^{\text {tw }}$ is an Ishibashi state obtained from the twisted sector of the $\mathbb{Z}_{2^{-}}$ orbifold theory. After modular transformation, the transition amplitude between two 
such twisted boundary states acquires the form

$$
\begin{aligned}
& Z_{++}=Z_{--}(q)=Z_{|D 0\rangle}(q)+Z^{\mathrm{tw}}(q)=\operatorname{tr}\left(\frac{1+g}{2} q^{H_{o p}}\right), \\
& Z_{+-}=Z_{-+}(q)=Z_{|D 0\rangle}(q)-Z^{\mathrm{tw}}(q)=\operatorname{tr}\left(\frac{1-g}{2} q^{H_{o p}}\right),
\end{aligned}
$$

where $g$ is the non-trivial element of $\mathbb{Z}_{2}$ acting on both Chan-Paton labels and oscillators. The twisted part $|D(z=0)\rangle\rangle^{\mathrm{tw}}$ of the boundary state is responsible for the term $Z^{\mathrm{tw}}(q)$ in the open string partition function in which the group element $g$ is inserted.

If we add the two boundary states $|D ;+\rangle$ and $|D ;-\rangle$ we obtain a boundary state without contributions from twisted sectors. This state comes with the regular representation of the orbifold group on Chan-Paton labels and it can be obtained directly by the projection method applied to the boundary state $|D(x=0)\rangle$ of the theory on the two-fold cover of the orbifold space. Turning the argument around, we see that boundary states at fixed points obtained with the projection method can be further decomposed by taking into account twisted sectors. This is the strategy we will now follow in our discussion of twisted boundary states for Gepner models.

\subsection{Twisted boundary states in the Gepner model}

In this section we will construct additional boundary states for the Gepner model, which are charged under the twisted sector states in short orbits of the orbifold group $\Gamma$. The method will be similar to the one used in flat space. The role of the state $|D(x=0)\rangle\rangle^{\text {tw }}$ is played Ishibashi states built from states in $K / 2$-twisted sector of the Gepner models. Our aim is to improve the boundary states associated with short orbits of $\Gamma$ by adding twisted sector Ishibashi states.

There are a few general statements one can make about this procedure whenever $\mathbb{Z}_{2}$ appears as the maximal stabilizer subgroup of the theory. In this case, the short orbits have length $K 2^{r-1}$. The application of the projection (9) leads to two equal pieces in the projected sum. Each of the two pieces can be interpreted as a sum over elements of the orbifold group modulo the stabilizer. It means that the boundary states we started with are not elementary but rather have to be replaced by a linear combination of the projected state and Ishibashi states from the $\nu=K / 2$ twisted sector, i.e.

$$
\left.\left|\Lambda_{s}, \Xi_{s}\right\rangle_{\text {proj }} \mapsto \beta\left|\Lambda_{s}, \Xi_{s}\right\rangle_{\text {proj }} \pm \sum_{\lambda_{s}, \mu_{s}} \alpha_{\lambda_{s}, \mu_{s}}\left|\lambda_{s}, \mu_{s}\right\rangle\right\rangle^{\mathrm{tw}}
$$

where and $\left.\left|\lambda_{s}, \mu_{s}\right\rangle\right\rangle$ is an Ishibashi state built on a short orbit primary labeled by $\lambda_{s}$ and $\mu_{s}$. The coefficients $\alpha$ and $\beta$ in the boundary state have to be chosen in such a way that the boundary state leads to a consistent open string spectrum. We will see that we can easily get information on $\beta$ from general considerations, whereas $\alpha$ is model dependent. 
Let us start the discussion by computing the open string spectrum in the case that one boundary state is given by the improved short orbit boundary state and the other one is given by a projected long orbit boundary state. In this case, we can get conditions on $\beta$ only, since the twist fields are orthogonal to all fields of the untwisted sector. The open string sector is determined by the fusion rules of the elements of the short orbit with those of the long orbit (cf. eq. (10) above). According to our general discussion following eq. (10), the overall multiplicity of the fields propagating in the open string sector is $2 \beta$. Therefore, minimal normalization suggests to pick

$$
\beta=\frac{1}{2} \text {. }
$$

Of course one has to check that this choice gives consistent open string spectra when tested against other short orbit states of the form (23). We will do this in the explicit examples below.

To prepare for the discussion of Gepner models, we determine the improved boundary states in a single $\mathcal{N}=2$ minimal model, where we mod out by the group generated by the current $(0,1,1)$. We pick a minimal model with $k=0 \bmod 4$. In this case, the length of a generic orbit of the current $(0,1,1)$ is $2 k+4$ and there is a short orbit for $l=k / 2$. As we outlined above, our plan is to add Ishibashi states $|k / 2, m+\nu, s+\nu\rangle\rangle^{\text {tw }}$ built on twisted sector fields to the short orbit boundary states. Our ansatz for the improved boundary states is

$$
\begin{aligned}
\left|\frac{k}{2}, M, S\right\rangle= & \frac{1}{2}\left|\frac{k}{2}, M, S\right\rangle_{\text {proj }}+ \\
& \left.+\tilde{\alpha} \frac{1}{2^{1 / 4}(k+2)} \sum_{\nu=0}^{k+1} \sum_{m, s}^{e v}(-1)^{\nu} e^{i \pi m \frac{M+\nu}{k+2}} e^{i \pi s \frac{S+\nu}{2}}\left|\frac{k}{2}, m, s\right\rangle\right\rangle^{\mathrm{tw}},
\end{aligned}
$$

where the sum over $m$ and $s$ is constrained to $m+s=$ even. We allow for an overall factor $\tilde{\alpha}$ in front of the twisted part of the boundary state. It will be adjusted later so that we get a consistent open string spectrum. To this end, we have to compute the transition amplitude between the twisted parts of the boundary state and to perform a modular transformation. This results in the following expression for the contribution $Z^{\text {tw }}$ of the twisted sector to the full open string partition function :

$$
Z^{\mathrm{tw}}=\tilde{\alpha}^{2} \frac{2}{k+2} \sum_{\nu=0}^{k+1} \sum_{l^{\prime}, m^{\prime}, s^{\prime}}^{e v}(-1)^{\nu} \sin \pi \frac{l^{\prime}+1}{2} \delta_{\nu+m^{\prime}+\frac{k+2}{2}\left(\nu+s^{\prime}\right)}^{(2 k+4)} \delta_{\nu+s^{\prime}}^{(2)} \chi_{l^{\prime}, m^{\prime} s^{\prime}}(q)
$$

From our previous discussion on the minimal normalization of $\beta$ we know that the untwisted part of the full partition function computed from the state (24) has a factor $1 / 2$ standing in front of each character. We pick $\tilde{\alpha}$ in such a way that there is a factor of $1 / 2$ in front of the twisted contribution as well. This means that we take $\tilde{\alpha}$ to be

$$
\tilde{\alpha}^{2}=\frac{k+2}{4} \text {. }
$$


For this choice to be consistent, all characters in the full partition function should come with integer coefficients. The twisted sector gives negative contributions for $l^{\prime}=2 \bmod 4$, positive contributions for $l^{\prime}=0 \bmod 4$ and no contributions for $l^{\prime}$ odd. With our choice of $\tilde{\alpha}$, all contributing characters come with a factor $\pm 1 / 2$ in front.

From the untwisted part of the partition function we obtain characters with even $l^{\prime}$. As we mentioned before, their coefficient is $1 / 2$. Characters with odd $l^{\prime}$ are forbidden by the fusion rules. Putting everything together, we have shown that the sum of the twisted and the untwisted part of the partition function contains the characters with $l^{\prime}=0$ mod 4 with an integer coefficient. The characters with $l^{\prime}=2 \bmod 4$ get subtracted, so that they are not propagating in the open string sector.

Equation (26) for $\tilde{\alpha}$ admits the choice of a sign similar to the flat space case. In our general discussion, this corresponds to a character of the stabilizer subgroup. Since our orbifold group is $\mathbb{Z}_{2}$, the latter reduces to the choice of a sign. The open string partition function has an opposite sign in front of the twisted part in the case that the two boundary states have opposite signs. In this case, the characters with $l^{\prime}=0 \bmod 4$ get removed from the partition function and those with $l^{\prime}=2 \bmod 4$ survive. This can be summarized in the following formulas

$$
\begin{aligned}
& Z_{++}=Z_{--}=\sum_{\nu=0}^{k+1} \sum_{l^{\prime}, m^{\prime}, s^{\prime}}(-1)^{\nu} \delta_{l^{\prime}}^{(4)} \delta_{\nu+m^{\prime}+\frac{k+2}{2}\left(\nu+s^{\prime}\right)} \delta_{\nu+s^{\prime}}^{(2)} \chi_{l^{\prime}, m^{\prime}, s^{\prime}}(q) \\
& Z_{+-}=Z_{-+}=\sum_{\nu=0}^{k+1} \sum_{l^{\prime}, m^{\prime}, s^{\prime}}(-1)^{\nu} \delta_{l^{\prime}+2}^{(4)} \delta_{\nu+m^{\prime}+\frac{k+2}{2}\left(\nu+s^{\prime}\right)} \delta_{\nu+s^{\prime}}^{(2)} \chi_{l^{\prime}, m^{\prime}, s^{\prime}}(q) .
\end{aligned}
$$

Let us now discuss short orbit states in the full Gepner model. The length of a short orbit is $K / 2$. In the individual minimal models, this means that $l_{i}=k_{i} / 2$ in the case that $2 k_{i}+4$, the orbit length of the single minimal model, does not divide $K / 2$. In the case that $K / 2$ is a multiple of the orbit length of a minimal model, an arbitrary primary can be chosen in that particular model. If the number of minimal models, for which we need $l_{i}=k_{i} / 2$, is $r^{\prime}$, the factors in the minimal models can be ordered in such a way that these minimal models are the factors $1, \ldots, r^{\prime}$. This means that the labels of the short orbit boundary state are

$$
\Lambda_{s}=\left(\frac{k_{1}}{2}, \ldots, \frac{k_{r^{\prime}}}{2}, L_{r^{\prime}+1}, \ldots, L_{r}\right)
$$

with arbitrary labels $\Xi$. Our general discussion of short orbit states and the discussion of the single minimal model in this section motivates the following ansatz for a short orbit boundary state in the full Gepner model

$$
\left|\Lambda_{s}, \Xi_{s}\right\rangle=\frac{1}{2}\left|\Lambda_{s}, \Xi_{s}\right\rangle_{\text {proj }}+
$$




$$
\begin{gathered}
+\alpha c N \sum_{\nu=0}^{\frac{K}{2}-1} \sum_{\nu_{j}=0,1} \sum_{\mu} \sum_{l_{r^{\prime}+1}, \ldots, l_{r}}(-1)^{\nu}(-1)^{\frac{s_{0}^{2}}{2}} \prod_{j=r^{\prime}+1}^{r} \frac{\sin \left(l_{j}, L_{j}\right)_{k_{j}}}{\sqrt{\sin \left(l_{j}, 0\right)_{k_{j}}}} \times \\
\left.\times \prod_{j=1}^{r} e^{i \pi \frac{M_{j}+\nu}{k_{j}+2} m_{j}} e^{-i \pi \frac{s_{j}}{2}\left(S_{j}+\nu+2 \nu_{j}\right)} e^{-i \pi \frac{s_{0}}{2}\left(S_{j}+\nu-2 \sum \nu_{j}\right)}\left|\frac{k_{1}}{2}, \ldots, \frac{k_{r^{\prime}}}{2}, l_{r^{\prime}+1}, \ldots, l_{r} ; \mu\right\rangle\right\rangle^{\mathrm{tw}} \\
\text { where } \quad c=\left(\sqrt{2} \prod_{j} \sqrt{\sqrt{2}\left(k_{j}+2\right)}\right)^{-1}
\end{gathered}
$$

and $N=1 / \sqrt{K 2^{r}}$. To determine the prefactor $\alpha$ of the twisted part of the boundary state, we compute the transition amplitude between the boundary state and itself and modular transform to the open string sector. This leads to the following result for the twisted part of the partition function

$$
\begin{aligned}
Z^{\mathrm{tw}}= & \alpha^{2} \frac{1}{2} \frac{1}{K 2^{r}} \sum_{\nu} \sum_{\nu_{j}} \sum_{\lambda^{\prime}, \mu^{\prime}}(-1)^{\nu} \delta_{\nu-2 \sum \nu_{j}+s_{0}^{\prime}-2}^{(4)} \\
& \prod_{j=1}^{r^{\prime}} \frac{2}{k_{j}+2} \sin \pi \frac{l_{j}^{\prime}+1}{2} \delta_{\substack{\left(2 k_{j}+4\right) \\
\nu+m_{j}^{\prime}+\frac{\left(k_{j}+2\right)}{2}\left(\nu+2 \nu_{j}+s_{j}^{\prime}\right)}} \delta_{\nu+2 \nu_{j}+s_{j}^{\prime}}^{(2)} \\
& \prod_{j=r^{\prime}+1}^{r} N_{L_{j} L_{j}}^{l_{j}^{\prime}} \delta_{\nu+m_{j}^{\prime}}^{\left(2 k_{j}+4\right)} \delta_{2 \nu_{j}+\nu+s_{j}^{\prime}}^{(4)} \chi_{\lambda^{\prime}, \mu^{\prime}}(q) .
\end{aligned}
$$

The computation for the factors $1, \ldots, r^{\prime}$ is like that for the short orbits in a single minimal model and the computation for the other factors works exactly as the computation for the projected boundary states of long orbits.

Given that we have characters with a prefactor of $1 / 2$ in the untwisted part of the partition function, the consistent choice for $\alpha$ is

$$
\alpha= \pm \sqrt{K 2^{r-r^{\prime}-1} \prod_{j=1}^{r^{\prime}}\left(k_{j}+2\right)}
$$

Again, there are two different boundary states $\left|\lambda_{s}, \Xi_{s} ; \pm\right\rangle$ depending on the sign chosen for $\alpha$. Adding the twisted and untwisted part of the partition function, we see that characters with an odd $l_{j}^{\prime}$ do not appear in both the untwisted and twisted part. Those with an even number of $l_{j}^{\prime}=2 \bmod 4$ add up and appear with multiplicity one in the total partition function. Those with an odd number of $l_{j}^{\prime}=2 \bmod 4$ appear with a negative sign in the twisted part of the partition function and get removed from the spectrum in the total partition function. 
In the case of the Gepner model, there is usually more than one short orbit, and to check the consistency of the boundary states constructed above we have to compute the partition functions for all combinations of boundary states. The short orbits differ in the choice of $L_{j}$ in the minimal model factors which are not restricted to be on short orbits and in the $\Xi_{s}$. The computation of the partition function for two different boundary states is not much different from that above. In the $S U(2)$ fusion coefficients, one of the $L_{j}$ is replaced by some other label $\tilde{L}_{j}$ for all $j=r^{\prime}+1, \ldots, r$. Furthermore, one has to substitute the sum $m_{j}^{\prime}+\nu$ with $m_{j}^{\prime}+\nu+M_{j}-\tilde{M}_{j}$ and similarly $s_{j}^{\prime}+\nu$ with $s_{j}^{\prime}+\nu+S_{j}-\tilde{S}_{j}$.

\section{Conclusions and Outlook}

In this paper, we have constructed A-type boundary states in the Gepner model, which cannot be obtained by the projection method. These boundary states contain Ishibashi states from twisted sectors. In particular, they carry RR-charge under the RR-fields in the twisted sector of the Gepner model. Geometrically, this means that the branes wrap exceptional cycles, i.e. cycles coming from the resolution of a singularity. It has been shown in examples [12] that the rank of the intersection matrix computed from projected boundary states equals the number of homology three-cycles that do not come from the resolution. This clearly demonstrates that the projection method is missing interesting boundary states whenever the corresponding CY-manifold develops singular curves. Some of these extra boundary states are now provided by our construction.

In this paper, we considered only A-type boundary states. B-type boundary states in Gepner models have been obtained in [5]. It is possible to reinterpret the formulas given there in terms of simple currents orbifolds.

For B-type boundary states, the RR-charges associated with $(a, c)$-fields become important and replace the $(c, c)$-fields in our discussion above. It is well known that mirror symmetry relates these two different types of bulk fields, i.e. the space of $(a, c)$-fields on the original 3-fold $M$ gets mapped into the $(c, c)$-fields on the mirror $W$ and vice versa. Let us recall that mirror symmetry of closed string theories can be understood as an orbifold construction [43] with the group

$$
\mathcal{G}=\left(\prod_{i=1}^{r} \mathcal{G}_{i}\right) / \Gamma^{\prime} \cong\left(\prod_{i=1}^{r} \mathbb{Z}_{k_{i}+2}\right) / \mathbb{Z}_{\frac{K}{2}}
$$

where $\mathcal{G}_{i}$ is the group generated by the element $\left(2 ; 0, \ldots, m_{i}=2, \ldots, 0 ; 0, \ldots, s_{i}=\right.$ $2, \ldots, 0)$ and $\Gamma^{\prime}$ is the intersection between the orbifold group $\Gamma$ and the product of groups $\mathcal{G}_{i}$ in the nominator. Most importantly, this orbifold construction allows to compute the bulk partition function for the theory on the mirror $W$.

Following our general discussion above, one can extend these constructions of the mirror theory to obtain A-type boundary states on $W$ through an orbifold procedure 
with the group $\Gamma \times \mathcal{G}$. Obviously, the resulting spectra in the open string sector will be organized in terms of orbits of this orbifold group. On the other hand, orbits of $\Gamma \times \mathcal{G}$ do show up in the explicit formulas for open string spectra of B-type boundary states in the string theory on the original 3-fold $M[5]$. The fact that the partition functions of $\Gamma \times \mathcal{G}$-projected (A-type) D-branes on $W$ coincide with those found in [5] for B-type branes on $M$ confirms that the orbifold description of mirror symmetry extends to open strings (cp. also $[2,6])$.

In the detailed analysis of the $\Gamma \times \mathcal{G}$-orbifold theories one finds a rich pattern of orbits with short orbits of various lengths appearing for a typical model. This signals the existence of additional boundary states which are not obtained by projection from the tensor product theory. In principal, these states can be constructed along the lines of Section 5 above. Only technically this becomes more involved due to the possibly complicated orbit structure. The resulting B-type boundary states on $M$ are charged under fields which arise from desingularization of singular points and of singular curves.

In $[10,12,11]$ B-type boundary states have been compared with vector bundles on Calabi-Yau manifolds, which are elliptic [10] or K3 [12, 11] fibrations. For short orbit states it was found that there are a number of vacua propagating. The interpretation of [10] was that these boundary states do not correspond to elementary branes. This is indeed supported by our analysis. The elementary short orbit states should contain a twisted part.

Let us remark that our techniques can also be applied to boundary states in bulk theories with a D-type modular invariant. Boundary states for such theories have been discussed recently in [8]. In the context of simple current constructions, D-even modular invariants can be constructed by modding out an additional $\mathbb{Z}_{2}$ current in a minimal model. The open string sector is then organized in orbits of this current.

The CFT-construction we have used in this work allow to obtain a large number of boundary states extending the set of states which were known before, if the underlying Calabi-Yau 3-fold possesses $\mathbb{Z}_{2}$-singularities along curves. It is interesting to point out once more that the geometric scenario of Section 4 suggests the existence of many other important D-brane states that are charged under $(c, c)$-fields from the $K / N$-twisted sectors when $N \neq 2$. The latter come with the desingularization of a $\mathbb{Z}_{N \neq 2^{-}}$-singularity over a curve $C$ on $M$. While boundary states charged under such $K / N$-twisted $(c, c)$-fields satisfy A-type gluing conditions for the diagonally embedded superconformal algebra, it would be inconsistent with the $\mathrm{U}(1)$ charges of the corresponding $(c, c)$-fields to require such A-type gluing conditions for each minimal model separately. But so far, all treatments of D-branes in Gepner models assumed the strong version of the gluing condition which preserves all the individual superconformal algebras. It remains an interesting open problem to relax this requirement and, in particular, to construct $D$-branes associated with the resolution of $\mathbb{Z}_{N \neq 2}$ singularities over curves $C$ in $M$. 
Acknowledgements: We would like to thank M. Bianchi, R. Blumenhagen, M. Douglas, H. Liu, A. Recknagel, C. Römelsberger, R. Schimmrigk, Y. Stanev and S. Theisen for very useful discussions. V.S. is grateful to the Rutgers string group for their hospitality. I.B. thanks the II. Institut für Theoretische Physik for supporting her stay at Hamburg. We also want to thank A. Recknagel for his helpful remarks on the manuscript. 


\section{References}

[1] K. Becker, M. Becker, and A. Strominger, "Five-branes, membranes and nonperturbative string theory," Nucl. Phys. B456 (1995) 130-152, hep-th/9507158.

[2] H. Ooguri, Y. Oz, and Z. Yin, "D-branes on Calabi-Yau spaces and their mirrors," Nucl. Phys. B477 (1996) 407-430, hep-th/9606112.

[3] R. Minasian and G. Moore, "K-theory and Ramond-Ramond charge," JHEP 11 (1997) 002, hep-th/9710230.

[4] E. Witten, "D-branes and K-theory," JHEP 12 (1998) 019, hep-th/9810188.

[5] A. Recknagel and V. Schomerus, "D-branes in Gepner models," Nucl. Phys. B531 (1998) 185, hep-th/9712186.

[6] M. Gutperle and Y. Satoh, "D-branes in Gepner models and supersymmetry," Nucl. Phys. B543 (1999) 73, hep-th/9808080.

[7] M. Gutperle and Y. Satoh, "D0-branes in Gepner models and N = 2 black holes," Nucl. Phys. B555 (1999) 477, hep-th/9902120.

[8] M. Naka and M. Nozaki, "Boundary states in Gepner models," hep-th/0001037.

[9] I. Brunner, M. R. Douglas, A. Lawrence, and C. Römelsberger, "D-branes on the quintic," hep-th/9906200.

[10] D.-E. Diaconescu and C. Römelsberger, "D-branes and bundles on elliptic fibrations," hep-th/9910172.

[11] P. Kaste, W. Lerche, C. A. Lutken, and J. Walcher, "D-branes on K3-fibrations," hep-th/9912147.

[12] E. Scheidegger, "D-branes on some one- and two-parameter Calabi-Yau hypersurfaces," hep-th/9912188.

[13] M. R. Douglas and G. Moore, "D-branes, quivers, and ALE instantons," hep-th/9603167.

[14] A. N. Schellekens and S. Yankielowicz, "Extended chiral algebras and modular invariant partition functions," Nucl. Phys. B327 (1989) 673.

[15] A. N. Schellekens and S. Yankielowicz, "Simple currents, modular invariants and fixed points," Int. J. Mod. Phys. A5 (1990) 2903-2952. 
[16] J. Fuchs and C. Schweigert, "Orbifold analysis of broken bulk symmetries," Phys. Lett. B447 (1999) 266, hep-th/9811211.

[17] J. Fuchs and C. Schweigert, "Symmetry breaking boundaries. I: General theory," hep-th/9902132.

[18] J. Fuchs and C. Schweigert, "Symmetry breaking boundaries. II: More structures, examples," hep-th/9908025.

[19] L. Birke, J. Fuchs, and C. Schweigert, "Symmetry breaking boundary conditions and WZW orbifolds," hep-th/9905038.

[20] L. R. Huiszoon, A. N. Schellekens, and N. Sousa, "Open descendants of non-diagonal invariants," hep-th/9911229.

[21] G. Pradisi, A. Sagnotti, and Y. S. Stanev, "The open descendants of nondiagonal SU(2) WZW models," Phys. Lett. B356 (1995) 230-238, hep-th/9506014.

[22] G. Pradisi, A. Sagnotti, and Y. S. Stanev, "Completeness conditions for boundary operators in 2d conformal field theory," Phys. Lett. B381 (1996) 97-104, hep-th/9603097.

[23] D. Gepner, "Exactly solvable string compactifications on manifolds of SU(N) holonomy," Phys. Lett. B199 (1987) 380-388.

[24] D. Gepner, "Space-time supersymmetry in compactified string theory and superconformal models," Nucl. Phys. B296 (1988) 757.

[25] R. Blumenhagen and A. Wisskirchen, "Exactly solvable $(0,2)$ supersymmetric string vacua with GUT gauge groups," Nucl. Phys. B454 (1995) 561-586, hep-th/9506104.

[26] B. R. Greene, "String theory on Calabi-Yau manifolds," hep-th/9702155.

[27] E. Witten, "Phases of $\mathrm{N}=2$ theories in two dimensions," Nucl. Phys. B403 (1993) 159-222, hep-th/9301042.

[28] W. Boucher, D. Friedan, and A. Kent, "Determinant formulae and unitarity for the $\mathrm{N}=2$ superconformal algebras in two-dimensions or exact results on string compactification," Phys. Lett. B172 (1986) 316.

[29] A. B. Zamolodchikov and V. A. Fateev, "Disorder fields in two-dimensional conformal quantum field theory and $\mathrm{N}=2$ extended supersymmetry," Sov. Phys. JETP 63 (1986) 913-919. 
[30] P. D. Vecchia, J. L. Petersen, M. Yu, and H. B. Zheng, "Explicit construction of unitary representations of the $\mathrm{N}=2$ superconformal algebra," Phys. Lett. B174 (1986) 280.

[31] S. Nam, "The Kac formula for the $\mathrm{N}=1$ and the $\mathrm{N}=2$ superconformal algebras," Phys. Lett. B172 (1986) 323.

[32] S. Hosono, A. Klemm, and S. Theisen, "Lectures on mirror symmetry," hep-th/9403096.

[33] S. Katz, D. R. Morrison, and M. R. Plesser, "Enhanced gauge symmetry in type II string theory," Nucl. Phys. B477 (1996) 105-140, hep-th/9601108.

[34] M. Lynker and R. Schimmrigk, "A-D-E quantum Calabi-Yau manifolds," Nucl. Phys. B339 (1990) 121-157.

[35] J. Fuchs, A. Klemm, C. Scheich, and M. G. Schmidt, "Spectra and symmetries of Gepner models compared to Calabi- Yau compactifications," Ann. Phys. 204 (1990) 1-51.

[36] V. V. Batyrev, "Dual plyhedra and mirror symmetry for Calabi-Yau hypersurfaces in toric varieties," J. Alg. Geom. 3 (1994) 493-535, alg-geom/9310003.

[37] C. Clemens and P. Griffiths, "The intermediate jacobian of the cubic threefold," Annals of Math. 95 (1972) 281-356.

[38] D.-E. Diaconescu and J. Gomis, "Fractional branes and boundary states in orbifold theories," hep-th/9906242.

[39] F. Hussain, R. Iengo, C. Nunez, and C. A. Scrucca, "Aspects of D-brane dynamics on orbifolds," Fortsch. Phys. 47 (1999) 293, hep-th/9711020.

[40] M. Billo, B. Craps, and F. Roose, "On D-branes in type 0 string theory," Phys. Lett. B457 (1999) 61, hep-th/9902196.

[41] I. Brunner, R. Entin, and C. Römelsberger, "D-branes on T(4)/Z(2) and T-duality," JHEP 06 (1999) 016, hep-th/9905078.

[42] M. Oshikawa and I. Affleck, "Boundary conformal field theory approach to the critical two-dimensional Ising model with a defect line," Nucl. Phys. B495 (1997) 533 , cond-mat/9612187.

[43] B. R. Greene and M. R. Plesser, "Duality in Calabi-Yau moduli space," Nucl. Phys. B338 (1990) 15-37. 